\title{
THE ROCHLIN INVARIANT OF SURGERED, SEWN LINK EXTERIORS
}

\author{
MARK R. WOODARD
}

(Communicated by Frederick R. Cohen)

\begin{abstract}
One method of producing 3-manifolds is the sewing-up construction of W. R. Brakes. This involves identifying the boundary components of the exterior of a 2-component link. Under certain assumptions, this process yields homology handles which can be surgered to obtain homology spheres. The main result is a formula for the Rochlin invariant of homology spheres obtained by this construction when the link is proper.
\end{abstract}

\section{INTRODUCTION}

W. R. Brakes [B1, B2], produced a class of 3-manifolds via the following construction: Let $L=\left\{K_{1}, K_{2}\right\}$ be a 2-component link in a Z-homology 3-sphere. Then, since the exterior of $L$ has two components, one can "sew up" the link exterior by identifying the boundary components. Under appropriate assumptions, the manifolds obtained by this method are homology handles. These can then be surgered to produce homology spheres. This method yields a homology handle when $\beta n^{2}-2 n+\gamma= \pm 1$, where $\beta, \gamma$ come from the gluing matrix and $n=l k\left(K_{1}, K_{2}\right)$. We will denote the sewn-up link exterior by $S(L, A)$, and a surgered, sewn-up link exterior by $S S\left(L, A, \omega, \ell_{0}\right) . M\left(K_{1}, K_{2}, A\right)$ will denote the result of gluing two knot exteriors via $A$, that is, identifying the boundary components of the exteriors of two knots in disjoint homology spheres.

In this paper, a formula is given for the Rochlin invariant of homology spheres obtained by Brakes's method, for the case of a proper link. This generalizes the formula of Gordon [G] for glued-knot exteriors.

\section{Preliminaries}

All work that follows takes place in the smooth category. Let $M$ be a $\mathrm{Z}$ homology 3-sphere. Let $L=\left\{K_{1}, K_{2}\right\}$ be an oriented 2-component link. $L$

Received by the editors August 15, 1989 and, in revised form, January 10, 1990; the results of this paper were presented at the 851st meeting of the AMS in Hoboken, New Jersey, October 22, 1989.

1980 Mathematics Subject Classification (1985 Revision). Primary 57N10, 57M25. 
is proper if $l k\left(K_{1}, K_{2}\right)$ is even. We will assume that each $K_{i}$ comes equipped with a tubular neighborhood $N\left(K_{i}\right)$ and a longitude-meridian pair $\left(\ell_{i}, m_{i}\right)$, in which $\ell_{i}$ is a preferred longitude. Let $g: \partial N\left(K_{1}\right) \rightarrow \partial N\left(K_{2}\right)$ be an orientation-reversing diffeomorphism. Then $g$ is characterized by a matrix $A$ with integer entries as follows: If $g$ sends the homology class $\left[\ell_{1}\right]$ to the class $\left[\alpha \ell_{2}+\gamma m_{2}\right]$ and the class $\left[m_{1}\right]$ to the class $\left[\beta \ell_{2}+\delta m_{2}\right]$, then $A=\left[\begin{array}{ll}\alpha \beta \\ \gamma & \delta\end{array}\right]$. Since $g$ is a diffeomorphism, $\operatorname{det}(A)= \pm 1$, but $g$ is orientation-reversing, so $\operatorname{det}(A)=-1$.

The sewing-up construction of Brakes [B1] is carried out as follows: Identify points on $\partial\left(N\left(K_{1}\right)\right)$ with points on $\partial\left(N\left(K_{2}\right)\right)$ via the map $g$ corresponding to the matrix $A$. The closed manifold obtained from $E(L)$ under the identification will be denoted $S(L, A)$.

\section{FUNDAMENTAL GROUP AND HOMOLOGY}

Brakes [B2] has shown that

$$
H_{1}(S(L, A) ; \mathbf{Z})=\mathbf{Z} \oplus\langle\psi, \phi \mid \alpha n \psi+(\gamma-n) \phi=0 ;(\beta n-1) \psi+\delta \phi=0\rangle
$$

Here $\psi$ and $\phi$ are represented by meridians on $N\left(K_{1}\right)$ and $N\left(K_{2}\right)$. Note that when $n=0, H_{1}(S(L, A) ; \mathbf{Z}) \cong \mathbf{Z} \oplus \mathbf{Z}_{\gamma}$.

A G-homology handle is a 3-manifold with the same $G$-homology groups as $S^{1} \times S^{2}$. From the above presentation for $H_{1}(S(L, A) ; \mathbf{Z})$, it is clear that $S(L, A)$ is a Z-homology handle exactly when the matrix $\left[\begin{array}{cc}\alpha n & \gamma-n \\ \beta n-1 & \delta\end{array}\right]$ has determinant \pm 1 . This occurs when $\beta n^{2}-2 n+\gamma= \pm 1$.

Moreover, $S(L, A)$ is a $\mathbf{Z}_{2}$-homology handle exactly when this matrix has an odd determinant. Note that $\beta n^{2}-2 n+\gamma$ is odd if and only if $\gamma$ is odd, since $n$ is even.

Kawauchi [K1] has demonstrated a way to turn any homology handle into a homology sphere via surgery. For the sewn-up link construction, Brakes [B2] describes this as follows: Choose a path $\omega$ in $E(L)$ between $(0,0) \in \partial\left(N\left(K_{1}\right)\right)$ and $(0,0) \in \partial\left(N\left(K_{2}\right)\right)$. Let $T$ be a tubular neighborhood of $\omega$ chosen in such a way that $T \cap \partial\left(N\left(K_{i}\right)\right)=D_{i}^{2} \quad(i=1,2)$ are two 2-disks which are identified in $S(L, A)$. Thus, if $p: E(L) \rightarrow S(L, A)$ is the quotient map, $p(T)$ is a solid torus in $S(L, A)$. Let $m_{0}$ be a meridian on $p(T)$ and $\ell_{0}$ be any longitude meeting $m_{0}$ once. Form $\overline{S(L, A)-p(T)} \cup W$, where $W$ is a solid torus and the gluing identifies a meridian of $W$ with $\ell_{0}$. The result of this Dehn surgery will be denoted $S S\left(L, A, \omega, \ell_{0}\right)$. When the link is split, $S(L, A)=M\left(K_{1}, K_{2}\right) \# S^{1} \times S^{2}$, and it is clear that the surgery simply kills the $S^{1} \times S^{2}$ term as long as $\omega$ is chosen to intersect the splitting sphere in one point. 


\section{DeCOMPOSITION OF MATRICES}

AND A FRAMED LINK DESCRIPTION OF $S(L, A)$

Any $2 \times 2$ matrix $A=\left[\begin{array}{ll}\alpha & \beta \\ \gamma & \delta\end{array}\right]$ with determinant -1 can be "decomposed" in the following manner:

$$
A=\left[\begin{array}{ll}
-1 & 0 \\
m_{k} & 1
\end{array}\right]\left[\begin{array}{ll}
0 & 1 \\
1 & 0
\end{array}\right]\left[\begin{array}{cc}
-1 & 0 \\
m_{k-1} & 1
\end{array}\right]\left[\begin{array}{ll}
0 & 1 \\
1 & 0
\end{array}\right] \cdots\left[\begin{array}{ll}
0 & 1 \\
1 & 0
\end{array}\right]\left[\begin{array}{ll}
-1 & 0 \\
m_{1} & 1
\end{array}\right],
$$

or, equivalently,

$$
A^{\prime}=\left[\begin{array}{ll}
\gamma & \delta \\
\alpha & \beta
\end{array}\right]=\left[\begin{array}{ll}
m_{k} & 1 \\
-1 & 0
\end{array}\right]\left[\begin{array}{cc}
m_{k-1} & 1 \\
-1 & 0
\end{array}\right] \ldots\left[\begin{array}{cc}
m_{1} & 1 \\
-1 & 0
\end{array}\right] .
$$

The proof is due to Gordon [G].

In [H1], Hoste demonstrates a method for obtaining a framed link description of $S(L, A)$ for $L \subset S^{3}$. We will summarize his work here.

Let $\sqsubset K_{1}, K_{2} \sqsupset$ be shorthand notation for $S(L, A)$, and let $K_{1}^{\prime} \rightarrow K_{2}^{\prime}$ be notation for the 3-manifold obtained by gluing together knot exteriors via the matrix $A$. The slash on the arrow is to indicate that the knots $K_{1}^{\prime}$ and $K_{2}^{\prime}$ sit in different copies of $S^{3}$.

The meaning of $K_{1}^{\prime} \underset{A}{\longrightarrow} K_{1}, K_{2} \underset{B}{\longrightarrow} K_{2}^{\prime}$ and

should now be clear.

$$
B\left[\begin{array}{c}
K_{1}, K_{2} \\
K_{1}^{\prime}, K_{2}^{\prime}
\end{array}\right] A
$$

Lemma 3.1 [H1].

(a) $K_{1} \stackrel{A}{\rightarrow} \bigcirc \stackrel{B}{\rightarrow} K_{2}$ is homeomorphic to $K_{1} \stackrel{B\left[\begin{array}{ll}0 & 1 \\ 1 & 0\end{array}\right] A}{\longrightarrow} K_{2}$.

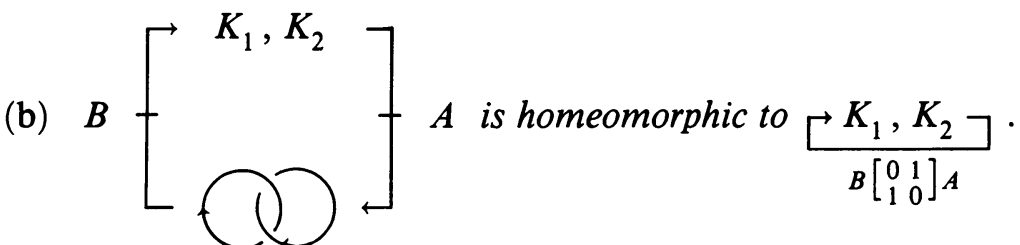

Proof. The exterior of the Hopf link is homeomorphic to torus $\times I$. This product structure induces the homeomorphism $J=\left[\begin{array}{ll}0 & 1 \\ 1 & 0\end{array}\right]$ between the tori which make up $\partial(E$ (Hopf link $))$. Q.E.D.

If one knew how to obtain a framed link description for $S(L, A)$ in the case $A=\left[\begin{array}{cc}-1 & 0 \\ m & 1\end{array}\right]$ and knew how to obtain a framed link description for glued knot exteriors when $A=\left[\begin{array}{cc}-1 & 0 \\ m & 1\end{array}\right]$, then Lemma 3.1 together with the matrix decomposition would indicate how to proceed in general.

Hoste shows that for the glued knot exterior case and $A$ as above, the framed link diagram may be obtained by band-connect summing the two knots with a framing of $-m$. For the sewn-up link exterior case, the knots are also bandconnect summed, but the band passes geometrically once through a 0 -framed unknot. The framing on the other knot is $-m+2 l k\left(K_{1}, K_{2}\right)$. 


\section{THE ROCHLIN INVARIANT: STATEMENT OF MAIN THEOREM}

Eells and Kuiper [E-K] (see also [H-N-K]) defined an invariant $\mu(M) \in \mathbf{Q} / \mathbf{Z}$ of $\mathbf{Z}_{2}$-homology 3-spheres. It can be proved that any $\mathbf{Z}_{2}$-homology 3-sphere bounds a 4-manifold $W$ so that $H_{1}(W ; \mathbf{Z})$ has no 2-torsion, and the quadratic form of $W$ (the intersection form on $H_{2}(W ; \mathbf{Z})$ ) is even. Then

$$
\mu(M) \equiv-\frac{\sigma(W)}{16} \quad(\bmod 1)
$$

where $\sigma(W)$ is the signature of the quadratic form of $W$. Since this is well defined by Rochlin's theorem [Ro], this invariant is often called the Rochlin invariant.

It has also been demonstrated [C-S] that $\mu(M)$ may be computed directly from a framed link diagram for $M$. Here

$$
\mu(M)=\frac{C^{2}-\sigma(T)}{16}+\operatorname{Arf}(C) \quad(\bmod 1)
$$

where $C$ is a characteristic sublink and $T$ is the linking matrix for the diagram. $C^{2}$ means the self-linking of the characteristic sublink. A characteristic sublink is one so that $C \cdot x \equiv x \cdot x(\bmod 2)$ for all components $x$ of the framed link.

In 1965 Robertello [Rob] defined an invariant of a knot in $S^{3}$. Gordon [G] generalized this to an invariant of a knot in a Z-homology sphere, and Kawauchi [K2] generalized it still further to an invariant of a proper link in a $\mathbf{Z}_{2}$-homology 3-sphere. The definition we will need is for a proper link in a Z-homology 3-sphere.

Let $(M, L)$ be a proper link in a $\mathbf{Z}$-homology 3 -sphere. Suppose $W$ is a 4-manifold with $H_{1}(W)=0$ and $F$ is a locally flat (possibly disconnected) surface of total genus zero in $W$. We say that $(W, F)$ is admissible for $(M, L)$ if $\partial(W, F)=(M, L)$ and if $B=[F] \in H_{2}(W, \partial W ; \mathbb{E})$ is characteristic for the quadratic form of $W$. That is $B \cdot z \equiv z^{2}(\bmod 2)$ for all $z \in H_{2}(W ; \mathbf{Z})$. (Note that $H_{2}(W ; \mathbf{Z}) \cong H_{2}(W, \partial W ; \mathbf{Z})$ since $H_{1}(\partial W ; \mathbf{Z})=0$.) The Robertello invariant $\rho(L)$ is now defined by

$$
\rho(L)=\frac{B^{2}-\sigma(W)}{16}-\mu(M) \in \mathbf{Q} / \mathbf{Z} .
$$

The possible values for $\rho(L)$ are 0 and $1 / 2$. For the case of a knot, Gordon [G] has shown that $\rho(K)=\operatorname{Arf}(K)$, the Arf invariant of the knot. Also, Kawauchi [K2] has shown that $\rho(L)=\rho\left(K_{0}\right)$ where $K_{0}$ is obtained from $L$ by band-connect summing the components. But by definition, $\operatorname{Arf}(L)=\operatorname{Arf}\left(K_{0}\right)$ [H2]. Thus we have $\rho(L)=\rho\left(K_{0}\right)=\operatorname{Arf}\left(K_{0}\right)=\operatorname{Arf}(L)$; so, for a proper link, the Robertello invariant is just a geometric version of the Arf invariant.

In preparation for the main theorem, consider the following construction. Let $V$ be a 4-manifold with boundary $S(L, A) \cup-M, H_{1}(V ; \mathbf{Z}) \cong \mathbf{Z}$ and 


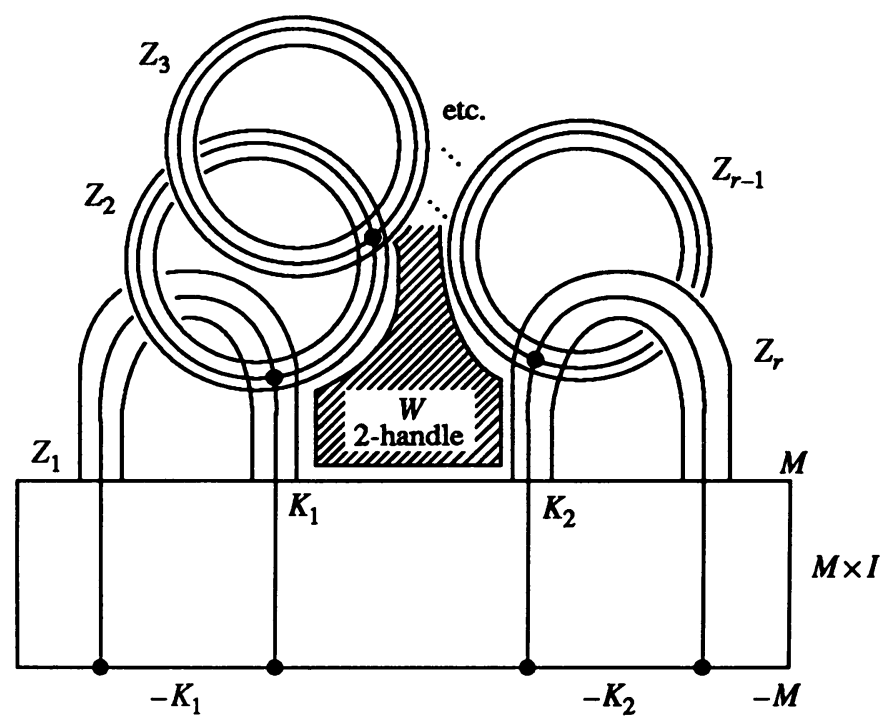

FIGURE 4.1

$H_{2}(V ; \mathbf{Z}) \cong \oplus_{i=1}^{r} \mathbf{Z}$ with intersection numbers

$$
z_{i} \cdot z_{j}= \begin{cases}-m_{i} & i=j \\ 1 & i=j \pm 1 \\ l k\left(K_{1}, K_{2}\right) & i=1, j=r \text { or } i=r, j=1 \\ 0 & \text { otherwise }\end{cases}
$$

Such a $V$ is constructed by decomposing the matrix $A$ as in $\S 3$, attaching two 2-handles to $M \times I$ along $N\left(K_{1}\right) \times 1$ and $N\left(K_{2}\right) \times 1$, and then attaching a string of plumbed $D^{2}$ bundles over $S^{2}$. The attachings are chosen to correspond to the integers in the decomposition, so that the boundary of $V$ is as desired (see Figure 4.1). See [G] for more details about this construction.

We want to perform surgery in $S(L, A) \subset \partial V$ to turn $S(L, A)$ into $S S\left(L, A, \omega, \ell_{0}\right)$. This is accomplished by adding a 2-handle along the loop $\omega$. The result is a 4 -manifold $V^{\prime}$ with $H_{1}\left(V^{\prime} ; \mathbf{Z}\right) \cong 0$ and $H_{2}(V ; \mathbf{Z}) \cong$ $H_{2}\left(V^{\prime} ; Z\right)$. This is seen by considering $V^{\prime}=V \cup B^{2} \times D^{2}$ and examining the long exact sequence of the pair $\left(V^{\prime}, V\right)$.

The following lemma is now easy:

Lemma 4.2. If $L$ is a proper link in a Z-homology sphere, then

$$
\mu\left(S S\left(L, A, \omega, \ell_{0}\right)\right)
$$

is independent of $\omega, \ell_{0}$. 
Proof. From the discussion above, we see that for a given $L$ and $A$, each $S S\left(L, A, \omega, \ell_{0}\right)$ bounds a 4-manifold with $H_{1}=0$ and the same quadratic form. Thus they all have the same $\mu$ invariant, independent of $\omega, \ell_{0}$. Note that the link must be proper for the quadratic form to be even. Q.E.D.

Our goal is to find a formula for the $\mu$ invariant of $S S\left(L, A, \omega, \ell_{0}\right)$ analogous to the formula given by Gordon for glued knot exteriors in [G].

We are now ready to state the main theorem:

Theorem 4.3. Let $L=\left\{K_{1}, K_{2}\right\}$ be a proper link in a Z-homology 3-sphere $M$. Let $A=\left[\begin{array}{ll}\alpha & \beta \\ \gamma & \delta\end{array}\right]$ be a matrix with determinant -1 . Let $n=l k\left(K_{1}, K_{2}\right)$ and assume $\beta n^{2}-2 n+\gamma$ is odd so that $S(L, A)$ is a $\mathbf{Z}_{2}$-homology handle. Then

$$
\begin{aligned}
\mu\left(S S\left(L, A, \omega, \ell_{0}\right)\right)=\delta(1-\alpha) \rho\left(K_{1}\right)+\alpha(1-\delta) \rho\left(K_{2}\right)+\alpha \delta \rho(L) \\
\\
+\mu(M)+\mu\left(S S\left(L^{n}, A\right)\right)+\alpha \delta \rho\left(L^{n}\right)(\bmod 1),
\end{aligned}
$$

where $L^{n}$ is the $(2,2 n)$ torus link.

These last terms can be computed by

Theorem 4.4. When $L^{n} \subset S^{3}$ is the $(2,2 n)$ torus link, we have

$$
\mu\left(S S\left(L^{n}, A\right)\right)+\alpha \delta \rho\left(L^{n}\right)=\mu(L(\gamma, \delta))+\left(\left(1-(-1)^{\alpha \delta}\right) n / 16\right)-(z(A, n) / 16),
$$

where $z(A, n) \in\{0, \pm 2\}$ will be defined in $\S 6$.

Corollary 4.5. We recover Gordon's formula [G] for glued knot exteriors. This follows because $S S\left(L^{0}, A\right)=L(\gamma, \delta)$ and because the Robertello invariant is additive for a split link $[\mathrm{Mu}]$. We have

$$
\begin{aligned}
& \mu\left(M\left(K_{1}, K_{2}, A\right)\right) \\
& \quad \delta \rho\left(K_{1}\right)-\alpha \delta \rho\left(K_{1}\right)+\alpha \rho\left(K_{2}\right)-\alpha \delta \rho\left(K_{2}\right) \\
& \quad+\alpha \delta \rho\left(K_{1}\right)+\alpha \delta \rho\left(K_{2}\right)+\mu(M)+\mu(L(\gamma, \delta)) \\
& =\delta \rho\left(K_{1}\right)+\alpha \rho\left(K_{2}\right)+\mu\left(M_{1}\right)+\mu\left(M_{2}\right)+\mu(L(\gamma, \delta))(\bmod 1),
\end{aligned}
$$

where $M=M_{1} \# M_{2}$. Q.E.D.

\section{Proof of Theorem 4.3}

The proof is somewhat analogous to the proof for glued knot exteriors given by Gordon [G]. The reader should be familiar with that proof.

Construct a 4-manifold $V^{\prime}$ with $\partial\left(V^{\prime}\right)=S S\left(L, A, \omega, \ell_{0}\right) \cup-M$ as in the previous section. Let $U$ be a simply connected 4-manifold with even quadratic form and $\partial(U) \cong S S\left(L, A, \omega, \ell_{0}\right)$. Let $H=V^{\prime} \cup-U$ along $S S\left(L, A, \omega, \ell_{0}\right)$. Then $H_{2}\left(H ; \mathbf{Z}_{2}\right) \cong H_{2}\left(V^{\prime} ; \mathbf{Z}_{2}\right) \oplus H_{2}\left(U ; \mathbf{Z}_{2}\right)$, since 
$S S\left(L, A, \omega, \ell_{0}\right)$ is a $\mathbf{Z}_{2}$-homology sphere. This fact also assures us that the quadratic form of $V^{\prime}$ over $Z_{2}$ is nonsingular and thus there exists a unique element $\bar{z} \in H_{2}\left(V^{\prime} ; \mathbf{Z}_{2}\right)$ so that $\bar{z} \cdot \bar{\theta} \equiv \bar{\theta}^{2}(\bmod 2)$ for all $\bar{\theta} \in H_{2}\left(V^{\prime} ; \mathbf{Z}_{2}\right)$ (see $[\mathrm{H}-\mathrm{N}-\mathrm{K}]$, p. 26). Also, since $U$ has an even quadratic form, $\bar{z} \cdot \bar{\theta}=0=\bar{\theta}^{2}$ for all $\bar{\theta} \in H_{2}\left(U ; \mathbf{Z}_{2}\right)$. Thus if $z \in H_{2}(H ; \mathbf{Z})$ is a class which reduces $\bmod 2$ to $\bar{z}$, then $z$ is characteristic for the quadratic form of $H$.

Now let $z=\sum_{i=1}^{r} a_{i} z_{i}, a_{i}=0$ or 1 . Each $z_{i}$ is represented by a 2 sphere for $i=2, \ldots, r-1$, while $z_{1}$ and $z_{r}$ are represented by 2-discs with boundaries $-K_{1}$ and $-K_{2}$, respectively. Let the surface which represents $z_{i}$ be denoted by $F_{i}$. We pipe these together to obtain a surface representing $z$ as in [G], although the case $a_{1}=1, a_{r}=1$ must be handled differently. Notice that in this case not all other $a_{i}$ 's are one (in fact $a_{1}=1$ implies that $a_{2}=0$, since $z$ is characteristic and $n$ is even), so there exists a $k$ with $a_{k}=0$. In this case, pipe together all of the surfaces for $i<k$ and pipe together those for $i>k$. The result is a surface $F$ such that

(1) if $a_{1}=a_{r}=0, F$ is a 2 -sphere in $\operatorname{int}(H)$.

(2) if $a_{1}=1$ and $a_{r}=0, F$ is a 2-disc with $\partial F=-K_{1} \subset-M$.

(3) if $a_{1}=0$ and $a_{r}=1, F$ is a 2-disc with $\partial F=-K_{2} \subset-M$.

(4) if $a_{1}=a_{r}=1, F$ is the union of two 2-discs which intersect $l k\left(K_{1}, K_{2}\right)$ times, and $\partial F=-L \subset-M$.

We will define an admissible pair $(W, B)$ for the four cases mentioned above. In case (1), let $H_{0}$ be obtained from $H$ by attaching a copy of $U_{1}$ along $M$. Here $U_{1}$ is a simply connected even 4-manifold with boundary $M$. Define $(W, B)=\left(H_{0}, F\right)-\left(E^{4}, E^{2}\right)$ where $\left(E^{4}, E^{2}\right)$ is a standard disc pair neighborhood of a point on $F$. For cases (2) through (4), simply let $(W, B)=(H, F)$.

Then $\partial(W, B)=$ : an unknot in $S^{3}$ (case 1$) ;-K_{1} \subset-M$ (case 2); $-K_{2} \subset$ $-M$ (case 3 ); $-L \subset-M$ (case 4 ). $B$ is characteristic for the quadratic form of $W$ by construction. Notice that for case (1) this statement uses the evenness of $U_{1}$.

Let $\partial(W, B)=(Y, J)$ so that $(Y, J)$ is the above for the four different cases. By definition,

$$
\rho(J)=\frac{z^{2}-\sigma(W)}{16}-\mu(Y) \quad(\bmod 1) .
$$

Define $b_{i}=1-a_{i}$. Then the following are easily verified by a case-by-case analysis:

$$
\begin{gathered}
\rho(J)=-a_{1} b_{r} \rho\left(K_{1}\right)-a_{r} b_{1} \rho\left(K_{2}\right)-a_{1} a_{r} \rho(L) ; \\
\mu(Y)=-\mu(M)+b_{1} b_{r} \mu(M) ; \\
\sigma(W)=\sigma(H)+b_{1} b_{r} \sigma\left(U_{1}\right) .
\end{gathered}
$$


Also,

$$
\sigma(H)=\sigma\left(V^{\prime}\right)-\sigma(U) \text { (by construction). }
$$

Working in $\mathbf{Q} / \mathbf{Z}$, we obtain the following:

$$
\begin{aligned}
\mu(S S & \left.\left(L, A, \omega, \ell_{0}\right)\right)=-\frac{\sigma(U)}{16}=\frac{\sigma(H)-\sigma\left(V^{\prime}\right)}{16} \quad \text { (substituting (5)) } \\
& =\frac{\sigma(W)-b_{1} b_{r} \sigma\left(U_{1}\right)-\sigma\left(V^{\prime}\right)}{16} \quad \text { (substituting (4)) } \\
& =\frac{\sigma(W)-z^{2}}{16}-\frac{b_{1} b_{r} \sigma\left(U_{1}\right)}{16}+\frac{z^{2}-\sigma\left(V^{\prime}\right)}{16} \\
& =-\rho(J)-\mu(Y)+b_{1} b_{r} \mu(M)+\frac{z^{2}-\sigma\left(V^{\prime}\right)}{16} \quad \text { (substituting (1)) } \\
& =a_{1} b_{r} \rho\left(K_{1}\right)+a_{r} b_{1} \rho\left(K_{2}\right)+a_{1} a_{r} \rho(L)+\mu(M)+\frac{z^{2}-\sigma\left(V^{\prime}\right)}{16}
\end{aligned}
$$

(substituting (2) and (3)).

Now $\sigma\left(V^{\prime}\right)$ depends only on the integers $m_{i}$ and $n=l k\left(K_{1}, K_{2}\right)$, and $z^{2}$ is determined by $m_{i}$ and $n$. Thus to figure out what $\frac{z^{2}-\sigma\left(V^{\prime}\right)}{16}$ is, we set $L=L^{n}$, the $(2,2 n)$ torus link. Thus $K_{1}=K_{2}=$ unknot in $S^{3}$, and $l k\left(K_{1}, K_{2}\right)=n$. Then $\rho\left(K_{1}\right)=\rho\left(K_{2}\right)=\mu(M)=0$, so $\frac{z^{2}-\sigma\left(V^{\prime}\right)}{16}+a_{1} a_{r} \rho(L)=$ $\mu\left(S S\left(L^{n}, A, \omega, \ell_{0}\right)\right)$. This term will be examined more closely in $\S 6$. It remains to be seen that $a_{1}=\bar{\delta}$ and $a_{r}=\bar{\alpha}$, which is proved by Gordon [G, $\mathrm{p}$. 162]. This result also requires $n$ to be even. Q.E.D.

\section{6. $\mu\left(S S\left(L^{n}, A\right)\right.$ : Proof of Theorem 4.4}

The idea will be to compare the link diagram picture of $L(\gamma, \delta)$ with that of $S S\left(L^{n}, A, \omega, \ell_{0}\right)$. Since the $\mu$ invariant does not depend on $\left(\omega, \ell_{0}\right)$, we will assume that $\omega$ is chosen as pictured below in Figure 6.1a. Moreover, if $\ell_{0}$ is chosen so that the framing on $\omega$ is 2 , we can blow down two components, yielding a 3-manifold with the same $\mu$ invariant as the one pictured in Figure 6.1b.

There is an obvious correspondence between the standard link diagram for a lens space $[R$, p. 272] and the one pictured above. The pictures differ only in the linking number of two components. Also, note that if $C$ is a characteristic sublink for the link diagram of $L(\gamma, \delta)$, then choosing the corresponding components in the diagram gives a characteristic sublink $C^{\prime}$ for $S S\left(L^{n}, A\right)$, since the linking numbers are the same $\bmod 2$. However, if $C$ contains both ends, then $C^{\prime 2}=C^{2}+2 n$, while if $C$ does not contain both ends, $C^{\prime 2}=C^{2}$.

Now let $T$ be the intersection matrix for $L(\gamma, \delta)$ and $T^{\prime}$ the intersection 


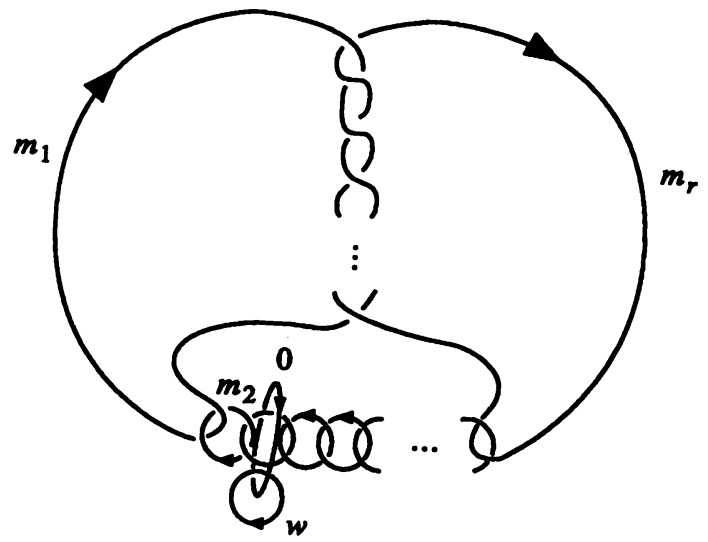

Figure 6.1a

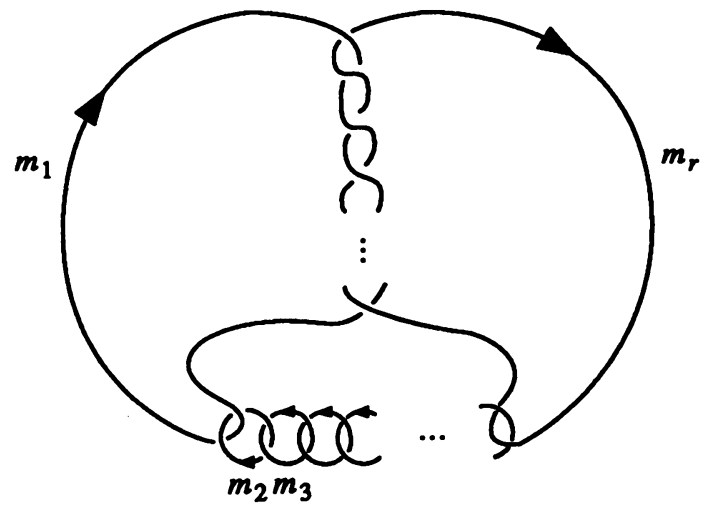

FIGURE 6.1b

matrix for $S S\left(L^{n}, A\right)$. Then $T$ and $T^{\prime}$ have the following form

$$
T=\left[\begin{array}{cccccc}
-m_{1} & 1 & 0 & \ldots & 0 & 0 \\
1 & -m_{2} & 1 & 0 & \ldots & 0 \\
0 & 1 & -m_{3} & 1 & 0 \ldots & \\
\vdots & \ddots & \ddots & \ddots & 0 & \\
0 & \ldots & & 0 & 1 & -m_{r}
\end{array}\right]
$$




$$
T^{\prime}=\left[\begin{array}{cccccc}
-m_{1} & 1 & 0 & \ldots & 0 & n \\
1 & -m_{2} & 1 & 0 & \cdots & 0 \\
0 & 1 & & & & \vdots \\
\vdots & \ddots & \ddots & & 0 & \\
0 & & & & & 1 \\
n & 0 & \ldots & 1 & & -m_{r}
\end{array}\right]
$$

It is clear that $\sigma(T)=\sigma\left(T^{\prime}\right) \pm\left\{\begin{array}{ll}0 \\ 2\end{array}\right.$ since after diagonalizing the matrices are identical except for one entry.

Lemma 6.3. The characteristic sublink for the framed link diagram for $S S\left(L^{n}, A\right)$ contains both ends if and only if both $\alpha$ and $\delta$ are odd.

Proof. Gordon [G] proves that $a_{1}=\bar{\delta}$ and $a_{r}=\bar{\alpha}$ when $C=\sum_{i=1}^{n} a_{i} z_{i}$ is characteristic, and the quadratic form is as in $T$ above. Q.E.D.

Thus, $C^{\prime 2}=C^{2}$ when either $\alpha$ or $\delta$ are even, but $C^{\prime 2}=C^{2}+2 n$ when both $\alpha$ and $\delta$ are odd. Thus $C^{\prime 2}=C^{2}+\left(1-(-1)^{\alpha \delta}\right) n$. Also, $\operatorname{Arf}(C)=0$, but $\operatorname{Arf}\left(C^{\prime}\right)=\alpha \delta \operatorname{Arf}\left(L^{n}\right)$.

It remains to compare $\sigma(T)$ and $\sigma\left(T^{\prime}\right)$. Let $m_{1}, \ldots, m_{r}$ be the integers in the decomposition of $A$. Let

$$
y_{r}=-m_{r}, \quad y_{r-1}=-m_{r-1}-\frac{1}{y_{r}}, \ldots, y_{1}=-m_{1}-\frac{1}{y_{2}} .
$$

(Without loss of generality, $m_{1} \neq 0$ and $m_{r} \neq 0$, by controlling the matrix decomposition in $\S 3$ ). Then when one diagonalizes $T$, the result is the diagonal matrix with $\left(y_{1}, \ldots, y_{r}\right)$ on the diagonal. However, when one diagonalizes $T^{\prime}$, the result is the matrix with $\left(z, y_{2}, \ldots, y_{r}\right)$ on the diagonal where

$$
z=y_{1}-\frac{n^{2}}{y_{r}}-\frac{n^{2}}{y_{r}^{2} y_{r-1}}-\frac{n^{2}}{\left(y_{r} y_{r-1}\right)^{2} y_{r-2}}-\cdots-\frac{n^{2}}{\left(y_{r} \ldots y_{3}\right)^{2} y_{2}}+(-1)^{r+1} \frac{2 n}{y_{r} \ldots y_{2}} \text {. }
$$

Now, given $-m_{1}, \ldots,-m_{r}$, let

$$
z(A, n)=\left\{\begin{aligned}
0 & \text { if } y_{1} \text { and } z \text { have the same sign, } \\
-2 & \text { if } y_{1}>0 \text { and } z<0 \\
2 & \text { if } y_{1}<0 \text { and } z>0
\end{aligned}\right.
$$

Then $\sigma\left(T^{\prime}\right)=\sigma(T)+z(A, n)$. Now we have

$$
\begin{aligned}
\mu\left(S S\left(L^{n}, A\right)\right)+\alpha \delta \operatorname{Arf}\left(L^{n}\right) & =\frac{C^{\prime 2}-\sigma\left(T^{\prime}\right)}{16} \\
& =\frac{C^{2}+\left(1-(-1)^{\alpha \delta}\right) n-\sigma(T)-z(A, n)}{16} \\
& =\mu\left(L(\gamma, \delta)+\frac{\left(1-(-1)^{\alpha \delta}\right) n}{16}-\frac{z(A, n)}{16} .\right.
\end{aligned}
$$

It would be desirable to have an easier method of computing $z(A, n)$ or some nice characterization. The following theorem has some partial results toward understanding $z(A, n)$, although it does not cover all cases. 
Theorem 6.4. Assume $\delta, \alpha \neq 0$.

(1) If $\gamma / \delta$ is positive, then $z(A, n)=0$ or -2 , while if it is negative, then $z(A, n)=0$ or +2 .

(2) If $\gamma / \alpha$ is positive, then $z(A, n)=0$ or +2 , while if it is negative, then $z(A, n)=0$ or -2 .

(3) If $\alpha / \delta$ is positive, then $z(A, n)=0$.

Proof. (1) follows because $y_{1}=\gamma / \delta$. That is, when the matrix is diagonalized, $y_{1}$ is a continued fraction with entries determined by the decomposition, and the decomposition really is a continued-fraction decomposition for $\gamma / \delta$. Now if $y_{1}$ is positive, +2 is not a possibility for $z(A, n)$, while if $y_{1}$ is negative, -2 is not a possibility.

(2) is similar to (1), although the continued fraction must be turned around. Details are left to the reader. (3) follows immediately from (1) and (2), since $\alpha / \delta=(\gamma / \delta) /(\gamma / \alpha)$. Q.E.D.

\section{ACKNOWLEDGMENTS}

The author would like to thank his thesis advisor, Dr. Allan Edmonds, for guidance during the preparation of this work, which was part of his $\mathrm{Ph} . \mathrm{D}$. thesis. Thanks also go to the referee for some helpful suggestions.

\section{BIBLIOGRAPHY}

[B1] W. R. Brakes, Property $R$ and superslices, Quart. J. Math. 31 (1980), 263-281.

[B2] _ Sewing up link exteriors, London Math. Soc. Lecture Notes 48 (1982), 27-37.

[C-S] S. Cappell and J. Shaneson, Some new four manifolds, Ann. of Math. 104 (1976), 61-72.

[E-K] J. Eells and N. H. Kuiper, An invariant for certain smooth manifolds, Annali di Mat. puru et appl. 60 (1963), 93-110.

[G] C. McA. Gordon, Knots, homology spheres, and contractible 4-manifolds, Topology 14 (1975), 151-172.

[H-N-K] F. Hirzebruch, W. D. Neumann, and S. S. Koh, Differentiable manifolds and quadratic forms, Marcel Dekker, New York, 1971.

[H1] J. Hoste, Sewn-up r-link exteriors, Pacific J. Math. 112 (1984), 347-382.

[H2] - The Arf invariant of a totally proper link, Topology Appl. 18 (1984), 163-177.

[K1] A. Kawauchi, H-cobordism, I; the groups among three dimensional homology handles, Osaka J. Math. 13 (1976), 567-590.

[K2] _ On the Robertello invariants of proper links, Osaka J. Math. 21 (1984), 81-90.

[Mu] K. Murasugi, On the Arf invariant of links, Math. Proc. Cambridge Philos. Soc. 95 (1984), 61-69.

[Rob] R. Robertello, An invariant of knot cobordism, Comm. Pure Appl. Math. 18 (1965), 543555.

[Ro] V. A. Rochlin, New results in the theory of four-dimensional manifolds, Dokl. Akad. Nauk SSSR 84 (1952), 221-224.

[R] D. Rolfsen, Knots and links, Publish or Perish, Wilmington, DE, 1976.

Department of Mathematics, Furman University, Greenville, South Carolina 29613 